\title{
Cryptosporidium parvum, Cryptosporidium ryanae, and Cryptosporidium bovis in samples from calves in Austria
}

\author{
Katharina Lichtmannsperger ${ }^{1}$ (D) - Josef Harl ${ }^{2} \cdot$ Katharina Freudenthaler $^{1} \cdot$ Barbara Hinney $^{3} \cdot$ Thomas Wittek $^{1}$. \\ Anja Joachim ${ }^{3}$
}

Received: 9 July 2020 / Accepted: 7 October 2020 / Published online: 15 October 2020

(C) The Author(s) 2020

\begin{abstract}
Fecal samples of 177 calves of up to 180 days of age with diarrhea from 70 farms in Austria were examined to obtain information on the occurrence of Cryptosporidium species. Initially, all samples were examined by phase-contrast microscopy. Cryptosporidium-positive samples $(55.4 \% ; n=98)$ were screened by gp60 PCR, resulting in $68.4 \%(n=67)$. parvum-positive samples. The remaining 31 gp60-PCR-negative and the phase-contrast microscopy negative samples $(n=79)$ were screened by PCR targeting a $700 \mathrm{bp}$ fragment of the $18 \mathrm{~S}$ rRNA gene. Sequencing of the PCR products revealed the presence of $C$. parvum $(n=69)$, C. ryanae $(n=11)$, and $C$. bovis $(n=7)$. The latter two species have never been described in Austria. C. parvum-positive samples were genotyped at the gp60 gene locus, featuring four subtypes (IIaA15G2R1, IIaA21G2R1, IIaA19G2R1, IIaA14G1R1). The most frequently detected subtype IIaA15G2R1 $(n=52)$ was present in calves from 30 different farms. IIaA14G1R1 $(n=5)$ occurred on a single farm, subtype IIaA21G2R1 $(n=4)$ on two farms, and subtype IIaA19G2R1 $(n=4)$ on three farms. The results confirm the widespread occurrence of zoonotic C. parvum in diarrheic calves.
\end{abstract}

Keywords Protozoal infection $\cdot g p 60 \cdot 18 S \cdot$ Fecal consistency

\section{Introduction}

To date, 38 Cryptosporidium species are known, of which four-C. parvum, C. bovis, C. ryanae, and C. andersonican be found in cattle. Cryptosporidium parvum and C. bovis are responsible for over $90 \%$ of bovine infections (Feng et al. 2018; Widmer et al. 2020). Cryptosporidium parvum is associated with diarrhea in neonatal calves and intra-herd prevalence extends up to 100\% (Avendaño et al. 2018; Holzhausen et al. 2019; Thompson et al. 2017). Cryptosporidium bovis

Section Editor: Lihua Xiao

Katharina Lichtmannsperger

Katharina.Lichtmannsperger@vetmeduni.ac.at

1 University Clinic for Ruminants, Department for Farm Animals and Veterinary Public Health, University of Veterinary Medicine Vienna, Wien, Austria

2 Institute of Pathology, Department of Pathobiology, University of Veterinary Medicine Vienna, Wien, Austria

3 Institute of Parasitology, Department of Pathobiology, University of Veterinary Medicine Vienna, Wien, Austria and $C$. ryanae are primarily found in the feces of postweaned calves and $C$. andersoni in the abomasum of adult cattle (Ryan et al. 2014). Nevertheless, C. ryanae and C. bovis were isolated from pre-weaned diarrheic and healthy calves in certain areas of Sweden, China, and Sudan (Silverlås et al. 2010; Taha et al. 2017; Wang et al. 2011). Human cryptosporidiosis is primarily caused by $C$. parvum and C. hominis, and diarrheic and healthy calves are considered major reservoirs for human infections (Razakandrainibe et al. 2018; Ryan et al. 2014). Cryptosporidium oocysts are microscopically indistinguishable due to their similar size and shape. Therefore, molecular methods are indispensable for species differentiation (Ryan et al. 2014). Previous investigations focusing on the typing of $C$. parvum by sequencing a section of the $60-k D$ glycoprotein (gp60) gene in calves primarily report the occurrence of the subtype families IIa and IId with some geographical differences (Feng et al. 2018; Ryan et al. 2014). Subtype family IIa dominates in industrialized nations such as Italy (Díaz et al. 2018), the USA (Xiao et al. 2007), New Zealand (Abeywardena et al. 2012), and Austria (Lichtmannsperger et al. 2019). Subtype family IId was commonly reported from less industrialized countries (Ryan et al. 2014) such as Sudan (Taha et al. 2017), Malaysia (Muhid 
et al. 2011), Egypt (Amer et al. 2013), and China (Wang et al. 2011). Subtype IIaA15G2R1 has been described as the predominant subtype in symptomatic and asymptomatic calves worldwide (Feng et al. 2018; Holzhausen et al. 2019).

Differentiation of Cryptosporidium on species and subtype level is apparently lacking in Austria. The aim of this study was to determine the occurrence of different Cryptosporidium species and genotypes in calves with diarrhea less than 180 days of age. It was hypothesized that besides C. parvum, other Cryptosporidia species occur in feces of diarrheic calves in Austria.

\section{Material and methods}

\section{Sample collection and microscopic examination}

Farmers and veterinarians from all over Austria were contacted and asked to participate in the study. In total, 177 calves with diarrhea originating from 70 farms were included. The fecal samples used in this investigation were collected during the study on the occurrence of $C$. parvum and Giardia intestinalis in diarrheic calves in Austria (Lichtmannsperger et al. 2019). Samples were collected per rectum during a farm visit. All calves younger than 180 days of age with diarrhea (soft, liquid, or watery feces) were included and sampled once by the first author or the local veterinarians. The samples were transferred to the Institute of Parasitology at the University of Veterinary Medicine Vienna for immediate diagnostics. All samples were screened for Cryptosporidium spp. by phase-contrast microscopy (PCM) as described previously (Lichtmannsperger et al. 2019). In brief, sample purification was performed using the sodium-acetate-acetic formalin (SAF) method, and the pellet was resuspended in phosphate-buffered saline. The suspension was filled into the chamber of a disposable hemocytometer, and oocysts were counted using PCM with 200-fold magnification. The number of oocysts was given in oocysts per gram feces (opg).

\section{DNA extraction and PCR for genotyping}

DNA was extracted from all diarrheic fecal as described previously (Lichtmannsperger et al. 2019).

For the detection of all Cryptosporidium spp. except C. parvum, a nested PCR protocol was implemented to amplify a $700 \mathrm{bp}$ fragment of the nuclear $18 \mathrm{~S}$ rRNA gene (18S). For genotype analysis of $C$. parvum, a $450 \mathrm{bp}$ section of the gp60 gene was amplified. The $18 S$ PCR was performed on all PCM-negative and gp60-negative samples. The primers were designed based on complete or almost complete $18 \mathrm{~S}$ sequences of various apicomplexan parasites mined from NCBI GenBank (https://www.ncbi.nlm.nih.gov/genbank/). The quality of the primers was tested using AmplifX v.2.0.7. (Nicolas Julien; https://inp.univ-amu.fr/en/amplifx-managetest-and-design-your-primers-for-pcr) and Primer-BLAST implemented in NCBI GenBank. The primers (Table 1) are specific to the genus Cryptosporidium and do not amplify the $18 S$ of other apicomplexan parasites. The reaction volume $(25 \mu \mathrm{l})$ contained $1 \mu \mathrm{l}$ of genomic DNA template, $14.375 \mu \mathrm{l}$ nuclease free water, $5.0 \mu \mathrm{l}$ of $5 \mathrm{X}$ Green GoTaq ${ }^{\circledR}$ Reaction Buffer (Promega, USA), $2.0 \mu \mathrm{l}$ of $25 \mathrm{mM} \mathrm{MgCl}_{2}, 0.5 \mu \mathrm{l}$ of $10 \mathrm{mM}$ dNTP mix, $0.125 \mu \mathrm{l}$ GoTaq G2® Polymerase (5 U/ $\mu \mathrm{l}$, Promega), and $1.0 \mu$ leach of $10 \mathrm{mM}$ oligonucleotide primers (Table 1). For the second PCR round, $0.5 \mu l$ template from the previous PCR was used. The cycling protocol for both reactions included an initial cycle of $94^{\circ} \mathrm{C}$ for $2 \mathrm{~min}$, followed by 20 (nest 1) $/ 35$ (nest 2) cycles of $94^{\circ} \mathrm{C}$ for $30 \mathrm{~s}, 56^{\circ} \mathrm{C}$ for $60 \mathrm{~s}$, $72{ }^{\circ} \mathrm{C}$ for $60 \mathrm{~s}$, and a final extension of $72{ }^{\circ} \mathrm{C}$ for $5 \mathrm{~min}$.

The gp60 PCRs were carried out on all samples positive by phase-contrast microscopy as described previously (Lichtmannsperger et al.2019). In brief, $1 \mu$ l of genomic DNA was used in a $25 \mu \mathrm{l}$ reaction volume with $13.675 \mu$ of nuclease free water, $5.0 \mu \mathrm{l}$ of $5 \mathrm{X}$ Green GoTaq ${ }^{\circledR}$ Reaction
Table 1 Primers utilized in nested PCR reactions amplifying sections of the $18 S$ of Cryptosporidium spp. and the gp60 of Cryptosporidium parvum from fecal samples. The $18 \mathrm{~S}$ primers and the protocol were designed for this investigation; the implemented protocol for the detection of gp60 was described previously by Peng et al. (2001)

\begin{tabular}{|c|c|c|c|c|}
\hline & Primer & Primer sequence $\left(5^{\prime}-3^{\prime}\right)$ & $\begin{array}{l}\text { Amplicon size } \\
\text { (bp) }\end{array}$ & $\begin{array}{l}\text { Annealing } \\
\left({ }^{\circ} \mathrm{C}\right)\end{array}$ \\
\hline \multirow[t]{4}{*}{$18 S$} & $\begin{array}{l}\text { Crypto18S } \\
\text { F1 }\end{array}$ & $\begin{array}{l}\text { for: ACATATCATTCAAGTTTCTG } \\
\text { ACCTATC }\end{array}$ & 766 & 56 \\
\hline & $\begin{array}{l}\text { Crypto18S } \\
\quad \text { R1 }\end{array}$ & rev: TCTCATAAGGTGCTGAAGGAGT & & \\
\hline & $\begin{array}{l}\text { Crypto18S } \\
\quad \text { F2 }\end{array}$ & for: CAGCTTTAGACGGTAGGGTATTGG & 740 & 56 \\
\hline & $\begin{array}{l}\text { Crypto18S } \\
\quad \text { R2 }\end{array}$ & $\begin{array}{l}\text { rev: TAAGGTGCTGAAGGAGTAAG } \\
\text { GAAC }\end{array}$ & & \\
\hline \multirow[t]{4}{*}{ gp60 } & AL3531 & for: ATAGTCTCCGCTGTATTC & 850 & 56 \\
\hline & AL3534 & rev: GCAGAGGAACCAGCATC & & \\
\hline & AL3532 & for: TCCGCTGTATTCTCAGCC & 450 & 60 \\
\hline & AL3533 & rev: GAGATATATCTTGGTGCG & & \\
\hline
\end{tabular}


Buffer (Promega), $0.2 \mu \mathrm{l}$ of $25 \mathrm{mM}$ dNTPs, $3.0 \mu \mathrm{l}$ of $25 \mathrm{mM}$ $\mathrm{MgCl}_{2}, 0.125 \mu$ l of GoTaq ${ }^{\circledR} \mathrm{G} 2$ DNA Polymerase $(5 \mathrm{U} / \mu \mathrm{l})$, and $1 \mu \mathrm{l}$ each of 20 pmol oligonucleotide primers (Table 1). For the second PCR round, $0.5 \mu \mathrm{l}$ template from the previous PCR was used. The cycling protocol for both reactions included one cycle of $94{ }^{\circ} \mathrm{C}$ for $2 \mathrm{~min}$, followed by 30 cycles of $95^{\circ} \mathrm{C}$ for $50 \mathrm{~s}, 56^{\circ} \mathrm{C}$ (nest1) $/ 60{ }^{\circ} \mathrm{C}$ (nest 2) for $50 \mathrm{~s}, 65^{\circ} \mathrm{C}$ for $60 \mathrm{~s}$, and a final extension of $65^{\circ} \mathrm{C}$ for $5 \mathrm{~min}$.

PCR products were subjected to electrophoresis on $2.0 \%$ agarose gels and visualized with ultraviolet light (LumiBIS 1.4, DNR Bio-Imaging Systems Ltd., Israel).

\section{Sequencing of PCR products}

Purification and sequencing in both directions was done at LGC Genomics GmbH (Berlin, Germany). The raw forward and reverse sequences (and electropherograms) were carefully checked and aligned with Bioedit v.7.0.8.0 (Hall 1999). Sequences were subjected to BLAST (https://blast.ncbi.nlm. nih.gov/Blast) searches at NCBI GenBank to identify the respective $g p 60$ and $18 S$ variants. All sequences were deposited in NCBI GenBank under the accession numbers (18S: MT611069-MT611099; gp60: МT637080MT637083).

\section{Statistical analysis}

The data were organized using IBM $®$ SPSS $®$ Statistics Version 24 (IBM, New York, USA). Normal distribution was calculated using the Kolmogorov-Smirnov test. A chi ${ }^{2}$ test was implemented for the comparison of categorical variables (fecal consistency). The mean C. parvum, C. ryanae, and $C$. bovis shedding was only calculated with samples confirmed by $g p 60$ or $18 S$ PCR. The age differences in calves shedding C. parvum, C. ryanae, and C. bovis were analyzed by using a one-way ANOVA and the post hoc Bonferroni correction for multiple testing. Differences were considered statistically significant if $p \leq 0.05$.

\section{Results and discussion}

One to 10 animals were sampled per farm (median $=2$; mean $=2.5$ ). The fecal consistency of the diarrheic calves appeared soft $(n=72)$, liquid $(n=82)$, or watery $(n=23)$. The age ranged from 1 to 164 days $($ median $=12$; mean $=27)$. The average oocyst shedding $(n=98)$ was $1 \times 10^{5}$ opg (range $=$ $3.0 \times 10^{3}-3.0 \times 10^{7} ;$ median $\left.=1.0 \times 10^{6} ; \mathrm{SD}=3.0 \times 10^{6}\right)$ (Lichtmannsperger et al. 2019).

Previously, the widespread occurrence of Cryptosporidium spp. in diarrheic calves from Austria was described, but without differentiation at the species and subtype levels (Lichtmannsperger et al. 2019). All PCM-positive samples ( $n=98$ ) were screened using the gp60 PCR, of which $68.4 \%$ (67/98) yielded positive results. The remaining gp60-negative samples $(n=31)$ were screened for Cryptosporidium spp. using the $18 S$ PCR assay (see Fig. 1 for details). All gp60positive samples were further sequenced to determine C. parvum subtypes. Four subtypes (IIaA15G2R1, IIaA14G1R1, IIaA21G2R1, IIaA19G2R1) were detected.
Fig. 1 A total of 177 fecal samples from diarrheic calves were screened by phase-contrast microscopy (PCM). Samples were further analyzed by the C. parvum-specific $60 \mathrm{kD}-$ glycoprotein PCR (gp60) and the Cryptosporidium species-specific $18 S$ PCR. For the determination of C. parvum subtypes, the gp60 locus was used (Fail = sequencing unsuccessful). *Phase-contrast microscopy negative and $18 \mathrm{~S}$ positive $C$. parvum samples were further characterized using fragments of the gp 60 gene. "Double infection with $C$. bovis and C. ryanae in one sample

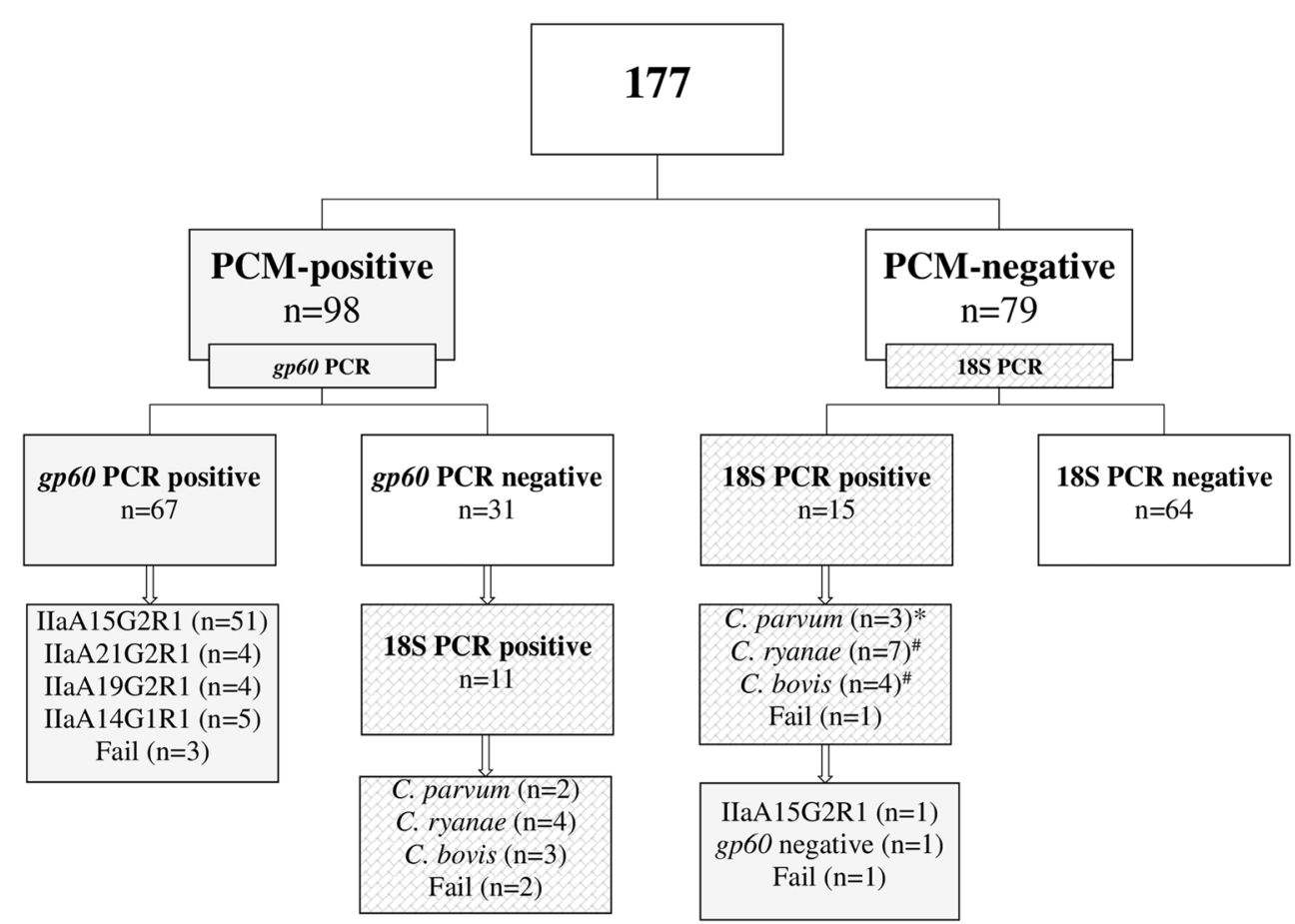


The most frequently detected subtype was IIaA15G2R1 $(n=$ $52)$ which was found on 30 farms. IIaA14G1R1 $(n=5)$ occurred on a single farm, subtype IIaA21G2R1 $(n=4)$ on two farms, and subtype IIaA19G2R1 $(n=4)$ on three farms. Subtype IIaA15G2R1 is the predominant subtype in symptomatic and asymptomatic calves worldwide, which was in accordance with our findings (Feng et al. 2018). A high subtype diversity but endemicity of a single subtype within herds or regions has previously been found in areas where animal movement is limited (Brook et al. 2009; Silverlås et al. 2010). Due to the implemented study design (sample size calculation, randomization), information concerning on-farm prevalence of subtypes is limited. Authors from Sweden reported similar observations of the on-farm-specific occurrence of $C$. parvum subtypes and assume that this was due to the dominating closed herd management systems (Silverlås et al. 2010). Closed herd management systems are common due to the small structured agriculture in Austria, which might be the reason for the similar results.

For the detection of further C. parvum species, the $18 \mathrm{~S}$ PCR assay was performed on all PCM-negative and all gp60-negative samples (Fig. 1). In total, $C$. parvum $(n=5)$, C. ryanae $(n=11)$, and $C$. bovis $(n=7)$ were detected. One sample contained both $C$. ryanae and C. bovis. The presence of both species in this sample was evident by double peaks in the electropherograms. Since the $18 S$ section analyzed was of same length in C. ryanae and C. bovis, the distinction of the two haplotypes was straightforward. Sequence analysis failed in four samples, which could be due to the presence of multiple Cryptosporidium strains.

The age of calves positive for $C$. parvum $(n=72)$ ranged from 3 to 127 days $($ mean $=14.5$; median $=10.0), 9$ to 126 (mean $=43.5$; media $n=35.0)$ for $C$. ryanae $(n=10)$, and 11 to 119 (mean $=64.6$; median $=60.0)$ for $C$. bovis $(\mathrm{n}=7)$. The age of the calves shedding $C$. parvum was significantly lower than from calves shedding $C$. bovis or $C$. ryanae $(p=0.00 ; p=$ 0.022). Between $C$. bovis and $C$. ryanae shedding calves, the age difference was not statistically significant $(p=0.866)$. The average number of opg shed by diarrheic calves was $1.7 \times 10^{6}$ $\left(\right.$ range $=5.0 \times 10^{3}$ to $2.6 \times 10^{7} ;$ median $\left.=4.4 \times 10^{5}\right)$ for C. parvum $(n=69), 1.1 \times 10^{4}$ (range: $2.5 \times 10^{3}$ to $2.3 \times 10^{4}$; median $\left.=10^{4}\right)$ for $C$. ryanae $(\mathrm{n}=4)$, and $1.3 \times 10^{4}$ (range $=$ $2.5 \times 10^{3}$ to $2.8 \times 10^{4}$; median $\left.=1.0 \times 10^{4}\right)$ for $C$. bovis $(\mathrm{n}=$ 3). Oocysts of $C$. bovis and C. ryanae were shed in lower numbers in comparison to $C$. parvum. However, the number of excreting animals was too low for statistical comparison.

Some authors report a higher prevalence of Cryptosporidium spp. when using light microscopy, which is in accordance with our findings (Taha et al. 2017). A potential explanation might be that some of the microscopypositive samples were wrongly classified due to the morphologically similar appearance of other particles such as yeast spores (Taha et al. 2017).
Genetic characterization of diarrheic fecal samples using the $18 S$ PCR assay showed the occurrence of $C$. ryanae and C. bovis in young diarrheic calves. The youngest animals were 9 and 11 days old, respectively. The results are in accordance with other studies, where $C$. bovis was found in calves from 5 days of age and C. ryanae from the second week of live (Åberg et al. 2019; Wang et al. 2011). Considering Cryptosporidium PCR results (gp60 or 18S; $n=93$ ), the occurrence of C. parvum (77.4\%), C. ryanae (11.8\%), and C. bovis $(7.5 \%)$ is comparable to an investigation from the Sudan on young ( $<6$ months) diarrheic calves, which featured C. parvum (73.5\%), C. ryanae (13.2\%), and C. bovis (1.8\%) (Taha et al. 2017). Additionally, C. andersoni was detected, which was not the case in the present study. Since only gp60negative samples were screened with the $18 S$ PCR assay, the occurrence of $C$. ryanae and $C$. bovis was likely underestimated in the present sample.

C. parvum occurred significantly more often $(p=0.007)$ in animals with liquid or watery diarrhea $(n=105)$ versus animals showing softened feces $(n=72)$. Cryptosporidium ryanae and $C$. bovis exclusively occurred in animals with soft $(n=8)$ respectively liquid $(n=9)$ fecal consistency; none of the animals showed watery diarrhea. Cryptosporidium bovis was discussed as potentially pathogenic in a study in Sweden, where $C$. bovis was found in diarrheic calves as the only pathogen (Silverlås et al. 2013). Another investigation found no association between the presence of diarrhea and C. bovis or C. ryanae shedding (Åberg et al. 2019).

The results show the common occurrence of the zoonotic species C. parvum and the host-specific C. ryanae and C. bovis in diarrheic calves in Austria. C. parvum-infected calves are shedding high numbers of oocysts which leads to severe environmental contamination and further transmission. Young calves suffering from liquid or watery diarrhea must be considered C. parvum shedders and therefore have the potential to cause human infection. Due to the implemented methodology, the simultaneous occurrence of Cryptosporidium species cannot be excluded. The number of $C$. bovis and C. ryanae positive samples was probably underestimated in the examined samples. Molecular methods such as genotypespecific or multiplex PCR procedures should shed more light on the occurrence of coinfections with different Cryptosporidium species or genotypes.

Acknowledgments The authors would like to thank the local veterinarians for their cooperation and the laboratory technicians (in particular Walpurga Wille-Piazzai) for their constructive work during this study.

Authors' contribution All authors jointly planned the study and analysis and approved the final version of the manuscript.

Funding Open access funding provided by University of Veterinary Medicine Vienna. The authors declare that they have no financial and personal relationships with other people or organizations that could 
inappropriately influence their work. The work of the veterinary undergraduate student (KF) was financially supported by the Austrian Association for Buiatrics (ÖBG). The sponsor did not participate in the study planning, conduct or data analysis, nor the manuscript compilation.

\section{Compliance with ethical standards}

This trial was evaluated and approved by the institutional Ethics and Animal Welfare Committee of the University of Veterinary Medicine Vienna.

Conflict of interest The authors declare that they have no conflict of interest.

Open Access This article is licensed under a Creative Commons Attribution 4.0 International License, which permits use, sharing, adaptation, distribution and reproduction in any medium or format, as long as you give appropriate credit to the original author(s) and the source, provide a link to the Creative Commons licence, and indicate if changes were made. The images or other third party material in this article are included in the article's Creative Commons licence, unless indicated otherwise in a credit line to the material. If material is not included in the article's Creative Commons licence and your intended use is not permitted by statutory regulation or exceeds the permitted use, you will need to obtain permission directly from the copyright holder. To view a copy of this licence, visit http://creativecommons.org/licenses/by/4.0/.

\section{References}

Åberg M, Emanuelson U, Troell K, Björkman C (2019) Infection dynamics of Cryptosporidium bovis and Cryptosporidium ryanae in a Swedish dairy herd. Vet Parasitol X:100010

Abeywardena H, Jex AR, Nolan MJ, Haydon SR, Stevens MA, McAnulty RW, Gasser RB (2012) Genetic characterisation of Cryptosporidium and Giardia from dairy calves. Infect Genet Evol 12:1984-1993

Amer S, Zidan S, Adamu H, Ye J, Roellig D, Xiao L, Feng Y (2013) Prevalence and characterization of Cryptosporidium spp. in dairy cattle in Nile River delta provinces, Egypt. Exp Parasitol 135:518523

Avendaño C, Ramo A, Vergara-Castiblanco C, Sánchez-Acedo C, Quílez J (2018) Genetic uniqueness of Cryptosporidium parvum from dairy calves in Colombia. Parasitol Res 117:1317-1323

Brook EJ, Hart CA, French NP, Christley RM (2009) Molecular epidemiology of Cryptosporidium subtypes in cattle in England. Vet J 179:378-382

Díaz P, Varcasia A, Pipia AP, Tamponi C, Sanna G, Prieto A, Ruiu A, Spissu P, Díez-Baños P, Morrondo P, Scala A (2018) Molecular characterisation and risk factor analysis of Cryptosporidium spp. in calves from Italy. Parasitol Res 117:3081-3090
Feng Y, Ryan U, Xiao L (2018) Genetic diversity and population structure of Cryptosporidium. Trends Parasitol 34:997-1011

Hall TA (1999) BioEdit: a user-friendly biological sequence alignment editor and analysis program for Windows 95/98/NT. Nucl Acid Symp Series 41:95-98

Holzhausen I, Lendner M, Göhring F, Steinhöfel I, Daugschies A (2019) Distribution of Cryptosporidium parvum gp60 subtypes in calf herds of Saxony, Germany. Parasitol Res 118:1549-1558

Lichtmannsperger K, Hinney B, Joachim A, Wittek T (2019) Molecular characterization of Giardia intestinalis and Cryptosporidium parvum from calves with diarrhea in Austria and evaluation of point-of-care tests. Comp Immunol Microbiol Infect Dis 66:101333

Muhid A, Robertson I, Ng J, Ryan U (2011) Prevalence of and management factors contributing to Cryptosporidium sp. infection in preweaned and post-weaned calves in Johor, Malaysia. Exp Parasitol 127:534-538

Peng MM, Matos O, Gatei W, Das P, Stantic-Pavlinic M, Bern C, Sulaiman IM, Glaberman S, Lal AA, Xiao L (2001) A comparison of Cryptosporidium subgenotypes from several geographic regions. J Eukaryot Microbiol 48:28-31

Razakandrainibe R, Diawara EHI, Costa D, Le Goff L, Lemeteil D, Ballet JJ, Gargala G, Favennec L (2018) Common occurrence of Cryptosporidium hominis in asymptomatic and symptomatic calves in France. PLoS Negl Trop Dis 12:e006355

Ryan U, Fayer R, Xiao L (2014) Cryptosporidium species in human and animals: current understanding and research needs. Parasitology 141:1667-1685

Silverlås C, Näslund K, Björkman C, Mattsson JG (2010) Molecular characterisation of Cryptosporidium isolates from Swedish dairy cattle in relation to age, diarrhoea and region. Vet Parasitol 169: 289-295

Silverlås C, Bosaeus-Reineck H, Näslund K, Björkman C (2013) Is there a need for improved Cryptosporidium diagnostics in Swedish calves? Int J Parasitol 43:155-161

Taha S, Elmalik K, Bangoura B, Lendner M, Mossaad E, Daugschies A (2017) Molecular characterization of bovine Cryptosporidium isolated from diarrheic calves in the Sudan. Parasitol Res 116:29712979

Thompson S, Hamilton CA, Hope JC, Katzer F, Mabbott NA, Morrison LJ, Innes EA (2017) Bovine cryptosporidiosis: impact, host-parasite interaction and control strategies. Vet Res 48(1):42

Wang R, Wang H, Sun Y, Zhang L, Jian F, Qi M, Ning C, Xiao L (2011) Characteristics of Cryptosporidium transmission in preweaned dairy cattle in Henan, China. J Clin Microbiol 49:1077-1082

Widmer G, Carmena D, Kváč M, Chalmers RM, Kissinger JC, Xiao L, Sateriale A, Striepen B, Laurent F, Lacroix-Lamandé S, Gargala G, Favennec L (2020) Update on Cryptosporidium spp.: highlights from the seventh international Giardia and Cryptosporidium conference. Parasite 27:14

Xiao L, Zhou L, Santin M, Yang W, Fayer R (2007) Distribution of Cryptosporidium parvum subtypes in calves in eastern United States. Parasitol Res 100:701-706

Publisher's note Springer Nature remains neutral with regard to jurisdictional claims in published maps and institutional affiliations. 